\title{
Effect of Foretinib on Matrix \\ Metalloproteinase-2 (MMP2) Expression in Glioblastoma
}

\section{Maryam Fotoohi, Nasrin Hadi, and Faezeh Namazi}

Medical Biotechnology Research Center, Ashkezar Branch, Islamic Azad University, Ashkezar, Yazd, Iran

\section{Abstract}

Background: The most malignant form of infiltrating astrocytoma, glioblastoma multiforme (GBM), is one of the most aggressive human cancers. Foretinib diminished GBM cell invasion by downregulating the expression of matrix metalloproteinase 2 (MMP2).

The study aimed to examine the anti-tumor activity of foretinib and to test its effect on MMP2 expression in T98 cells.

Materials and Methods: T98 cells were used as an experimental model of glioblas-

Corresponding Author:

Maryam Fotoohi;

Medical Biotechnology

Research Center, Ashkezar

Branch, Islamic Azad

University, Ashkezar, Yazd,

Iran

Tel: $+98-9130012262$

email: m.ft75@yahoo.com

Production and Hosting by

Knowledge E

(c) Maryam Fotoohi et al. This article is distributed under the terms of the

Commons Attribution License, which permits unrestricted use and redistribution provided that the original author and source are credited.

Editor-in-Chief:

Dr. Alireza Rafiei

\section{G OPEN ACCESS}

toma. The effect of foretinib on the expression of MMP2 was evaluated using quantitative real-time polymerase chain reaction. Thereafter, the effect of foretinib on the enzyme levels of MMP was examined by zymography. Statistical analyses were performed with GraphPad Prism software.

Results: A reduction in the expression of MMP2 was observed with an increase in the concentration of foretinib.

Conclusion: Foretinib treatment leads to downregulation of MMP-2 and has a positive effect on T98 cells. We believe that foretinib can be subjected to further clinical investigation to develop a treatment for GBM.

Keywords: Glioblastoma, foretinib, MMP2, T98.

\section{Introduction}

Glioblastoma multiforme (GBM) is a common and invasive form of brain cancer. It is a highly malignant tumor of the central nervous system (CNS) and is resistant to radiation therapy and chemotherapy [1]. GBM accounts for $45.2 \%$ of primary malignant brain and CNS tumors, $54 \%$ of all gliomas, and $16 \%$ of all primary brain and CNS tumors in the United States [2]. GBM is the most aggressive diffuse glioma of astrocytic lineage and corresponds to grade IV based on WHO classification [3]. Differences in the incidence and death rates for specific cancers based on race and ethnic groups as well as gender suggest potential identifiable biological and environmental variables. A higher incidence 
of GBM has been reported in men as compared to women [4-5]. Factors associated with GBM risk are prior therapeutic radiation, decreased susceptibility to allergy, immune factors, and immune genes [6]. GBM has a poor prognosis accompanied by very low relative survival estimates; only a few patients reaching long-term survival status of 2.5 years and less than $5 \%$ of patients survive for 5 years post-diagnosis [2]. Treatment of GBM includes maximal surgical resection followed by radiotherapy with concurrent and adjuvant chemotherapy. Regardless of the initial response, all patients experience disease relapse. Therefore, there is a pressing need to develop improved therapeutic options for GBM patients [7]. Foretinib is a multi-kinase inhibitor that targets MET, RON, $A X L, T I E-2$, and VEGFR2 receptors. Foretinib binds to the adenosine triphosphate moiety of its targets, resulting in kinase inhibition. Foretinib has been shown to inhibit tumor cell proliferation, invasion, and angiogenesis [8]. Foretinib is a potent inhibitor of ROS1 fusion kinases, which act as oncogenes in glioblastoma [9]. Matrix metalloproteinases (MMPs) are a family of proteolytic enzymes that are capable of degrading various components of the extracellular matrix. They are involved in virtually all stages of cancer progression. For example, they are involved not only in the process of tumor invasion and metastasis but also in processes such as proliferation, adhesion, migration, differentiation, and apoptosis [10]. The overexpression of MMP-2, MMP-3, and MMP-9 has been detected in various types of human cancers, such as esophageal cancer, gastric carcinoma, and ovarian and lung cancer, and has been closely associated with tumor progression and reduced survival [11]. Some pieces of evidence have indicated that MMP-2 expression and activation, in particular, is upregulated in glioblastoma and is associated with its rapid progression and malignancy [12]. Cancer cell lines are important tools to study genetic aberrations and molecular pathways in cancer. In this study, we used the T98 GBM cell line, which is widely used as a model for GBM studies. We thus examined whether foretinib influences MMP2 expression in T98 cells.

\section{Materials and Methods}

\subsection{Cell culture}

The T98 cell line was purchased from National Cell Bank of Iran (NCBI, Tehran, Iran) and cells were maintained at $37^{\circ} \mathrm{C}$ with $5 \% \mathrm{CO}_{2}$ in Dulbecco's Modified Eagle Medium (DMEM, Gibco, USA) supplemented according to NCBI recommendations. Foretinib was purchased from ChemieTek (ChemieTek, USA). To investigate the effects of foretinib on 
T98 cells, the cultured cells were exposed to different concentrations of foretinib ( $5 \mu \mathrm{M}$, $10 \mu \mathrm{M}, 20 \mu \mathrm{M}$, and $0 \mu \mathrm{M}$ as a control) for $24 \mathrm{~h}$.

\subsection{RNA isolation and Complementary DNA synthesis}

Total RNA was extracted from treated samples using TRizol reagent (Invitrogen, USA) according to the manufacturer's instructions. The quality of the extracted RNA was determined by measuring A260/A280 ratio using NanoDrop spectrophotometer (Thermo Scientific, Waltham, MA, USA). Complementary DNA (CDNA) synthesis was performed using a commercial kit (TaKaRa, Kusatsu, Shiga Prefecture, Japan) according to the manufacturer's protocol.

\subsection{Quantitative real-time polymerase chain reaction}

Primers were designed using the National Center for Biotechnology Information (NCBI) website and gene runner software (Table 1). The specificity of the primers was confirmed by the BLAST program. The quantitative real-time PCR reactions were performed in triplicates using an ABI PRISM 7500 instrument (Applied Biosystems, USA). Briefly, 20 $\mathrm{ng} \mathrm{I}^{-1}$ of cDNA product was added to $10 \mu \mathrm{l}$ of master mix composed of $0.5 \mu \mathrm{l}$ of forward primer, $0.5 \mu \mathrm{l}$ of reverse primer, $3 \mu \mathrm{l}$ of DEPC-treated water, and $5 \mu \mathrm{l}$ of SYBR premix ExTaq II (TaKaRa, Kusatsu, Shiga Prefecture, Japan). The reaction conditions were set at $95^{\circ} \mathrm{C}$ for $10 \mathrm{~min}$ followed by 40 cycles of $95^{\circ} \mathrm{C}$ for $15 \mathrm{~s}, 60^{\circ} \mathrm{C}$ for $10 \mathrm{~s}$ and $72^{\circ} \mathrm{C}$ for $20 \mathrm{~s}$. Real-time data were evaluated and represented based on the $2^{-{ }^{\Delta C T}}$ method [13].

TABLE 1: Sequences of the primers.

\begin{tabular}{l|l} 
Gene & Forward Primer \\
\hline MMP2 & CTTCCAAGTCTGGAGCGATGT \\
GAPDH & CCACTCCTCCACCTTTGACG \\
\hline
\end{tabular}

Reverse Primer
TACCGTCAAAGGGGTATCCAT
CCACCACCCTGTTGCTGTAG

Size (bp) 119 107

\subsection{Zymography}

Zymography is a technique that employs electrophoretic separation of proteins through a polyacrylamide gel containing a proteolytic substrate [14]. Equal amounts of protein were applied to polyacrylamide gels copolymerized with gelatin A (Sigma). After electrophoresis, the gels were stained with Coomassie Brilliant Blue. Areas of enzymatic activity appeared as clear bands over the dark background. 


\subsection{Statistical analysis}

The statistical analyses were carried out using GraphPad Prism, version 6.0 (GraphPad, San Diego, CA, USA). Normality was evaluated by the Kolmogorov-Smirnov test. The one-way ANOVA was used to compare the data. Data were expressed as the mean \pm SD. $P<0.05$ was considered to be statistically significant.

\section{Results}

\subsection{MMP2 expression}

The effect of foretinib on MMP2 expression was investigated. Real-time PCR results indicate that expression of MMP2 was decreased by foretinib in a concentration-dependent manner. We observed the highest decrease at $20 \mu \mathrm{M}$ of foretinib. In addition, the expression of MMP2 was significantly reduced at various concentrations of foretinib. MMP2 expression at $5 \mu \mathrm{M}$ of foretinib was significantly higher than that at $10 \mu \mathrm{M}$ and $20 \mu \mathrm{M}$ of foretinib $(\mathrm{P}<0.05$ and $\mathrm{P}<0.004$ respectively). These data suggest that foretinib reduced the mRNA levels of MMP2 (Figure 1).

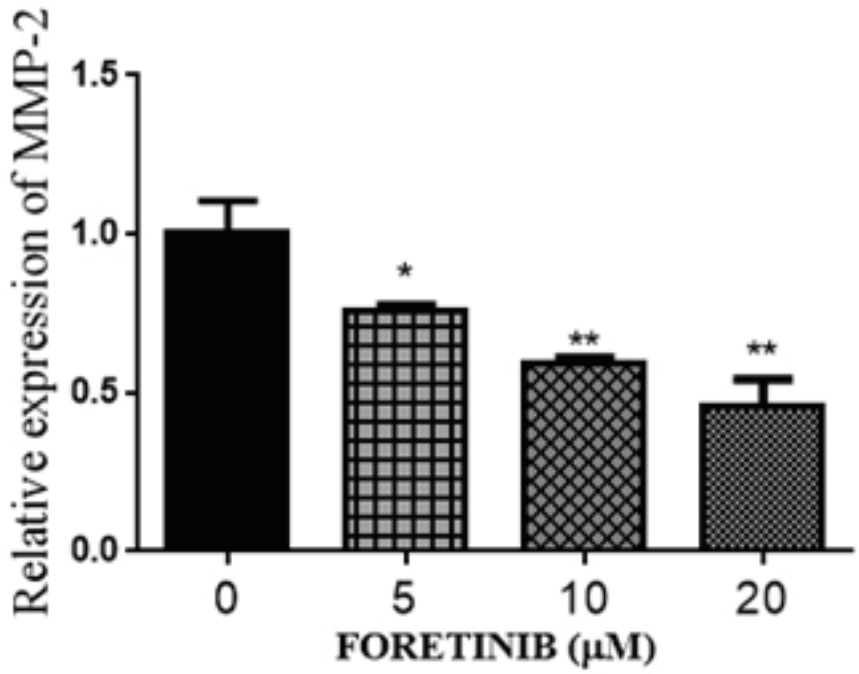

Figure 1: Decrease in MMP2 expression by increasing foretinib concentration. T98 cells were treated with increasing concentrations of foretinib $(0 \mu \mathrm{M}, 5 \mu \mathrm{M}, 10 \mu \mathrm{M}$, and $20 \mu \mathrm{M})$ and its effect on the expression of MMP-2 was determined as compared to control (*: $<0.05$ and $^{* *}:<0.004$, respectively). 


\subsection{Zymography}

MMP-2 is a putative therapeutic target to inhibit GBM invasion. According to the zymography results, foretinib reduced the enzyme levels of MMP-2 in a dose-dependent manner (Figure 2).
0
5
10
20

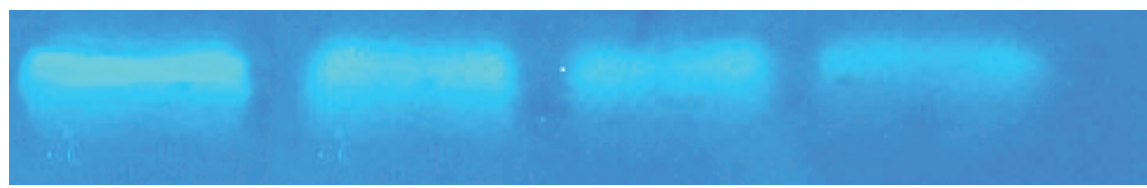

Figure 2: MMP-2 protein was purified and separated on a non-reducing polyacrylamide gel containing gelatin A. Gelatinolytic activities were visualized as clear bands against the blue background of stained gelatin. Foretinib (concentrations written in $\mu \mathrm{M}$ ) reduced the enzyme levels of MMP in a dose-dependent manner.

\section{Discussion}

In this study, we investigated the effect of foretinib as an oral multi-kinase inhibitor developed to primarily target the HGF/Met signaling pathway. Previously, foretinib has shown anti-cancer effects against some cancers in previous clinical researches. To explore the mechanism of anti-cancer properties of foretinib, the impact of this drug on the expression of MMP2 in GBM was assessed by qRT-PCR. We observed significant reduction in the expression of MMP2 by increasing the concentration of foretinib. It can be concluded that foretinib reduced the mRNA levels of MMP2. Based on the above findings, foretinib can be used as an effective drug for the treatment of GBM. However, it still requires further studies. Glioblastoma is a lethal malignant brain tumor and the current standard treatment of glioblastoma consists of surgical resection followed by concomitant chemoradiation therapy [15]. Foretinib is an oral drug which inhibits receptor tyrosine kinases c-Met and vascular endothelial growth factor receptor-2 (VEGFR-2). Both receptors have been implicated in the development and progression of cancer. Since foretinib is a multi-kinase c-Met/VEGFR-2 inhibitor, it targets c-Met and VEGFR-2 with high affinity but also inhibits platelet-derived growth factor- $\beta$ (PDGFR $\beta$ ), Tie-2, RON, c-Kit, and FLT3 kinases in vitro with low affinity [16]. Zillhardt et al. (2011) have investigated the effect of foretinib on metastasis in several ovarian cancer cell lines (CaOV3, CaOV-4, SKOV-3, and OVCAR-5) in a mouse model. This study has shown that foretinib blocks tumorigenesis and reduces invasive tumor growth in different models of ovarian cancer by affecting several critical tumor functions [16]. Nelson et al. (2013) suggested that 
adding foretinib to GBM treatment modes such as surgery and radiation may improve clinical outcomes by inhibiting the ability of glioblastoma cells to attack the normal brain tissue [17]. Human cancer cells might inherit numerous chromosomal aberrations and epigenetic alterations. Based on the interim integrative analysis of DNA copy number, gene expression, and DNA methylation aberrations in 206 GBM samples, the most common type of adult brain cancer, it has been observed that nucleotide sequence aberrations were present in 91 of the 206 GBMs [18]. On the other hand, one of the main challenges in GBM investigation is the identification of mechanisms used by GBM cells to invade the brain. With a variety of new pathway inhibitors and MMP inhibitors being recently introduced into clinical trials, the importance of discovering mechanisms of invasion that can be pharmacologically targeted is apparent. Overexpression of MMP2 is strongly correlated with astrocytic tumor grade and malignancy [12]. The extracellular proteases like MMP-2 allow the aggressive infiltration by degradation of the extracellular matrix (ECM) components and promoting GBM cell invasion. MMP2 expression levels correlate with malignant progression. Conversely, inhibition of MMP-2 activity reduces GBM invasion.[19-21] In conclusion, our data demonstrated that treatment with foretinib leads to downregulation of MMP2 in GBM cell line and has a positive effect on T98 cells . These findings suggest that foretinib has potential anti-glioma effects and can probably be used in chemotherapies as a treatment of GBM.

\section{Conflicts of Interest}

The authors declare no conflict of interest.

\section{References}

[1] Haas-Kogan D, et al. Protein kinase B (PKB/Akt) activity is elevated in glioblastoma cells due to mutation of the tumor suppressor PTEN/MMAC. Curr Biol. 1998:1195-S1.

[2] Ostrom QT, et al. CBTRUS statistical report: Primary brain and central nervous system tumors diagnosed in the United States in 2006-2010. Neuro Oncol. 2013;15.suppl_2:ii1-ii56.

[3] Louis DN, et al. The 2007 WHO classification of tumours of the central nervous system. Acta Neuropathol. 2007;114.2:97-109.

[4] McKinley BP, et al. The impact of age and gender on the incidence of glial tumors in New York state from 1976-1995. J Neurosurg. 2000;93.6:932-939. 
[5] Sloane D. Cancer epidemiology in the United States: racial, social, and economic factors. Cancer Epidemiol. 2009:65-83.

[6] Thakkar JP, et al. Epidemiologic and molecular prognostic review of glioblastoma. Cancer Epidemiol Biomarkers Prev. 2014;23.10:1985-1996.

[7] Messaoudi K, Clavreul A, Lagarce F. Toward an effective strategy in glioblastoma treatment. Part I: resistance mechanisms and strategies to overcome resistance of glioblastoma to temozolomide. Drug Discov Today. 2015;20.7:899-905.

[8] Chia SK, Ellard SL, Mates M, Welch S, Mihalcioiu C, Miller WH, et al. A phase-I study of lapatinib in combination with foretinib, a c-MET, AXL and vascular endothelial growth factor receptor inhibitor, in human epidermal growth factor receptor 2 (HER2)-positive metastatic breast cancer. Breast Cancer Res. 2017;19, no. 1:54.

[9] Chen HM, Tsai $\mathrm{CH}$, Hung WC. Foretinib inhibits angiogenesis, lymphangiogenesis and tumor growth of pancreatic cancer in vivo by decreasing VEGFR-2/3 and TIE-2 signaling. Oncotarget. 2015;6, no. 17:14940.

[10] Curran S, Murray Gl. Matrix metalloproteinases: molecular aspects of their roles in tumour invasion and metastasis. Eur J Cancer. 2000;36, no. 13 :1621-1630.

[11] González-Arriaga P, Pascual T, García-Alvarez A, Fernández-Somoano A, López-Cima MF, Tardón A. Genetic polymorphisms in MMP 2, 9 and 3 genes modify lung cancer risk and survival. BMC cancer. 2012;12, no. 1:121.

[12] Kesanakurti D, et al. Functional cooperativity by direct interaction between PAK4 and MMP-2 in the regulation of anoikis resistance, migration and invasion in glioma. Cell Death Dis. 2012;3.12:e445.

[13] Livak KJ, Schmittgen TD. Analysis of relative gene expression data using real-time quantitative PCR and the 2- $\Delta \Delta C T$ method. Methods, 2001. p. 402-408.

[14] Hawkes SP, Hongxia Li, Taniguchi GT. Zymography and reverse zymography for detecting MMPs and TIMPs. In Matrix metalloproteinase protocols, Humana Press, Totowa, NJ, 2010. p. 257-269.

[15] Jung TY, et al. Immunological characterization of glioblastoma cells for immunotherapy. Anticancer Res. 2013;33.6:2525-2533.

[16] Zillhardt M, et al. Foretinib (GSK1363089), an orally available multikinase inhibitor of c-Met and VEGFR-2, blocks proliferation, induces anoikis, and impairs ovarian cancer metastasis. Clinical Cancer Res. 2011;17.12:4042-4051.

[17] Nelson SK, et al. Et-070. Foretinib Inhibits Invasion, A Hallmark Of Malignancy, In Glioblastoma Multiforme. Neuro Oncol. 2013;15.suppl_3:iii37-iii61.

[18] Cancer Genome Atlas Research Network. Comprehensive genomic characterization defines human glioblastoma genes and core pathways. Nature. 2008;455.7216:1061. 
[19] Lu KV, Jong KA, Rajasekaran AK, Cloughesy TF, Mischel PS. Upregulation of tissue inhibitor of metalloproteinases (TIMP)-2 promotes matrix metalloproteinase (MMP)-2 activation and cell invasion in a human glioblastoma cell line. Laboratory investigation. 2004;84??:8.

[20] Badiga AV, et al. MMP-2 siRNA inhibits radiation-enhanced invasiveness in glioma cells. PloS One. 2011;6.6:e20614.

[21] Momeny $M$, et al. Blockade of vascular endothelial growth factor receptors by tivozanib has potential anti-tumour effects on human glioblastoma cells. Sci Rep. 2017;7:44075. 